\title{
Economic burden of unused medicines and its causes in households of Perinthalmanna region
}

\author{
Chandrasekhar Dilip*, Mohammed.S. Sayed, P.T. Sameer, P.K. ShesaMajeed, \\ Muhammed Kutty Shahanas \\ Department of Pharmacy Practice, Al Shifa College of Pharmacy, Perinthalmanna, Kerala, 679325, India
}

\section{A R T I C L E I N F O}

\section{Keywords:}

Unused medicines

Economic burden

Medication adherence

\begin{abstract}
A B S T R A C T
Background: The medical expenditure among the public in malabar region have been skyrocketing. The increased tendency to let the medicines unused ultimately leads to medicine wastage and huge economic loss. The purpose of this study was to assess the sheer bulk of unused medicines, factors responsible for their generation, and the cost bound to the wasted resource. The study also ventures further to identify the storage, handling and disposal practices of such unused medicines among the general public.

Methodology: A community based cross sectional descriptive study was carried out among 350 households of viii ${ }^{\text {th }}$ ward \& nearby wards. The self prepared questionnaire was incorporated. Questionnaire comprised of questions on medication taking behaviour, medication handling techniques including storage and disposal. The data collection form recorded the details of leftover medicines.

Results: The total economic burden of the population was computed to be rs.72,195.38, which sums up to rs.260 per household the major method of disposal was dumping (70.6\%). Solid dosage formulations were the most abundant dosage forms found unused (92\%). Storage conditions in households were satisfactory.

Conclusion: The evaluation of economic burden of unused medicines, utilization of medicines describes low medication adherence, high drug storage, higher leftover medicines and inappropriate use of medicines which suggests the need to educate the patients about proper and rational use of medicines. The fact that there are no proper disposal techniques for medicines points out the need of public awareness class on safe handling, storage and disposal of drugs in the households.
\end{abstract}

\section{Introduction}

The availability of medicines and their logistics have improved over the years in developing countries, especially with the introduction of essential drug programs. However, proper awareness on rational handling and the use of these medicines are inadequate in many developing countries. ${ }^{2}$

Consumption of medicines has increased over the years due to an increase in the availability of treatment for most diseases and the growing public interest in health and health products. The increasing disease burden especially in developing countries, desire for quick recovery from illness and the acceptance of self-medication among communities influences home storage of drugs. Challenges in healthcare delivery such as inadequate access, lack of medical personnel and frequent drug stock outs common in developing countries may influence communities to store drugs in homes. The patients at many times are not able to consume all the dispensed medications because of adverse events, alteration of health, medicines reaching expiry date. The challenges of having medicines in homes include poor storage as conditions such as humidity and temperature are not regulated. This increases the risk of deterioration and expiry of medicines. Due to lack of capacity to detect expired drugs in households; these medicines are in most cases taken by the residents, increasing the risk of adverse events. Controlling the use of drugs stored at home is a great task especially from unintentional users such as children which increases the risk of accidental poisoning. Presence of medicines at home has also been associated with sharing of drugs which further increase the risk of inappropriate drug use and hence the emergence of antimicrobial resistance. $^{4}$

Medicine use among Indians poses an entirely different scenario. Medicines are being purchased by patients in India with or without prescription and are stored in home. ${ }^{3}$ Storage of large quantity of medications at home increases the risk of a wide range of potential drug related problems, like

\footnotetext{
* Corresponding author. Dept of Pharmacy Practice, Alshifa college of Pharmacy, Poonthavanam P.0, Perinthalmanna, Kerala, 67932, India.

E-mail address: dillu7@gmail.com (C. Dilip).
} 
- Errors in taking medications

- Accidental poisoning

- Anti - microbial resistance

- Adverse drug reactions

- Medication sharing

- Drug abuse

- Waste of resources

- Environmental contamination

Factors influencing the use of medicines in the community include knowledge about use, the cost of medicines, regulatory systems, cultural factors, community beliefs and communication with prescribers among others. These factors play out at different levels including household and community. The way medicines are used is also influenced by individual beliefs about them, which may have been shaped by members of the immediate family and those in extended family networks at household level. The community is the immediate context in which individuals and families deal with their health problems. People talk to each other about therapies, creating and reinforcing existing medicine use cultures, and they rely on local sources of medicines. In order to address the problem of irrational use of medicines, health planners and administrators require specific information on the types, extent and the reasons of irrational use of medicines. ${ }^{5}$

The purpose of this study was to assess the sheer bulk of unused medicines, factors responsible for their generation, and the cost bound to the wasted resource. The study also ventures further to identify the storage, handling and disposal practices of such unused medicines among the general public.

\section{Methodology}

\subsection{Study design}

A community based descriptive, cross-sectional survey, conducted through face-to-face interviews using pre-validated structured questionnaire was carried out. The study was conducted from November 2017 to June 2018. The door to door self structured interview was carried out individually by the investigators. An average of $35 \mathrm{~min}$ was spent for a single household. The conversation equipped the investigator with the necessary particulars quintessential for filling the individual questionnaire and data collection form.

The study was initiated as part of a government project on drug waste management. It was named as "HOPE" in English and "PRATHYASHA" in Malayalam. It was then incorporated into the ongoing waste management program of the municipality, namely 'JEEVANAM'.

\subsection{Study population}

The study site in scrutiny is part of Malabar region of southern state of Kerala. The town of Perinthalmanna is a centre, bustling with healthcare organizations of both public and private sectors. A nonprobability sampling technique (convenience method) was employed and the eighth ward of Perinthalmanna municipality was chosen as the representative population. The choice was also backed by the population characteristics, support of the local governing body and overall acceptance of the public.

\subsection{Sample size}

A sample size calculation was performed using the Raosoft sample size calculator with a $5 \%$ confidence interval, $95 \%$ confidence level, the population size of 87356 (Population size of Perinthalmanna municipality according to the 2001 census Survey) The value was 383 . Since the study considers each household as a unit, the 351 households coming under the area of eighth ward was included in the study. A total of 1690 individuals were studied.

\subsection{Study tools}

A self prepared questionnaire, a data collection form and Morisky Medication Adherence Scale were used for the conduct of the study. The questionnaire was reviewed by experts and social workers for appropriateness. A door to door self structured interview was carried out individually by the investigators. An average of $35 \mathrm{~min}$ was spent for a single household. The conversation equipped the investigator with the necessary particulars quintessential for filling the individual questionnaire and data collection form. Questionnaire comprises of a series of questions regarding the demographics of the family members, presence of chronic illness, medication taking behaviour, their storage, handling and disposal. Data collection forms have been used to record the details pertaining to the unused/leftover drugs found in the particular household. A four item Morisky medication adherence scale was also employed in order to assess the medication adherence of the households under scrutiny. A pamphlet was designed and deployed in order to deliver brief details regarding the various aspects of drug use, handling, storage and disposal techniques.

\subsection{Data analysis}

The filled questionnaires were double-checked for accuracy and then the collected data were entered into an Excel spreadsheet. The data was then transferred to Statistical Package for Social Science (SPSS) version 23 for analysis. Descriptive statistics (descriptive, crosstab and chi-square) were used.

\section{Results}

The dataset consist of 1690 individuals, spread across 350 families. These families were residing at a particular area of the town, where the study was sanctioned by the local governing body. The total population was not directly involved, as only a fraction of them were present to answer the questionnaire at the time of interview. Non cooperating households were exempted from the study. $28.6 \%$ of the households had 5 family members, $68.6 \%$ of the families had $4-6$ members in total [Fig. 1]. Age of the family members was inclined towards the lower extreme. $10.4 \%$ were above 55 years of age compared to $35.3 \%$ below 18 years of age. The number of members in a family didn't affect the cost of unused medicines $\left(x^{2}=21.049, \mathrm{df}=27, \mathrm{p}=0.784\right)$. From the total sample population, $90 \%$ did not have any medical professional in their family, in contrast to $10 \%$ who had a medical professional in their family. The presence of a medical professional in the family, however reduced the cost due to unused medicines $\left(x^{2}=21.947, \mathrm{df}=9\right.$, $\mathrm{p}=0.003$ ).

$49.7 \%$ of the population was suffering from one or more than one form of chronic diseases which demand chronic medicine use such as

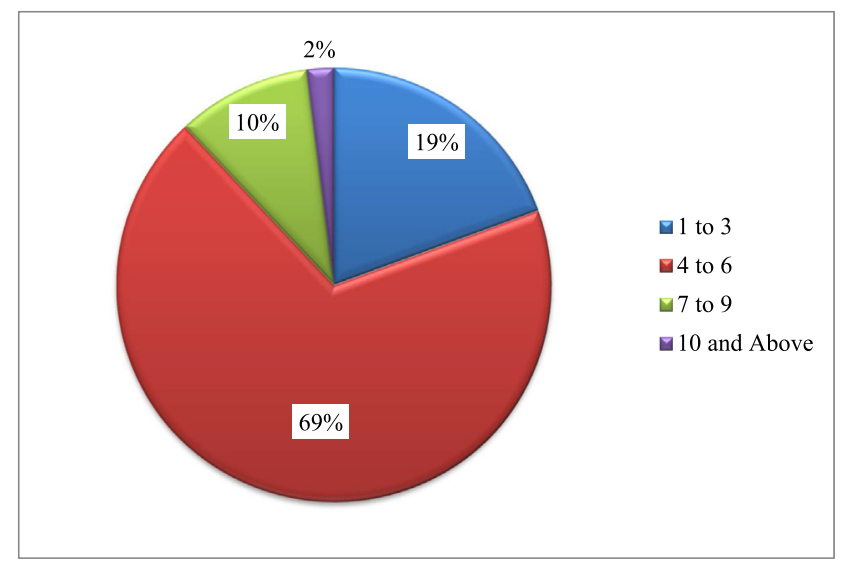

Fig. 1. Number of family members in a household. 


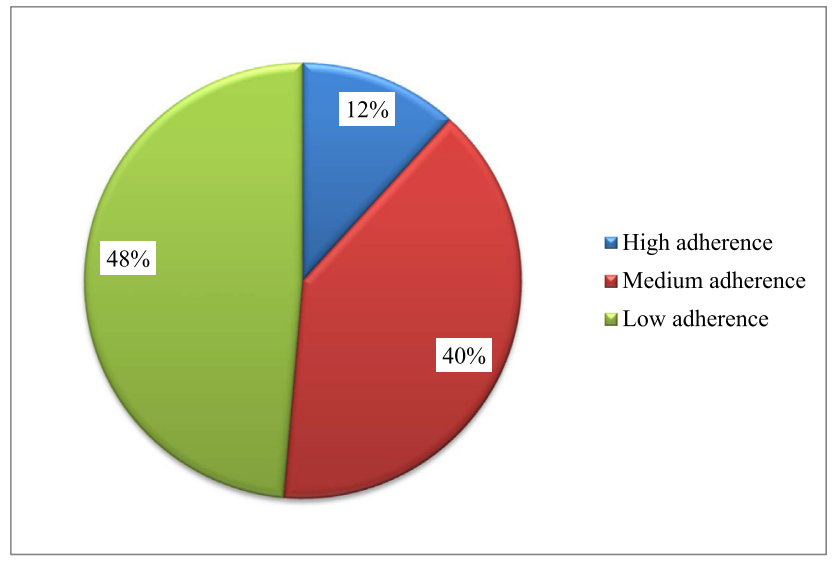

Fig. 2. Medication adherence of the population.

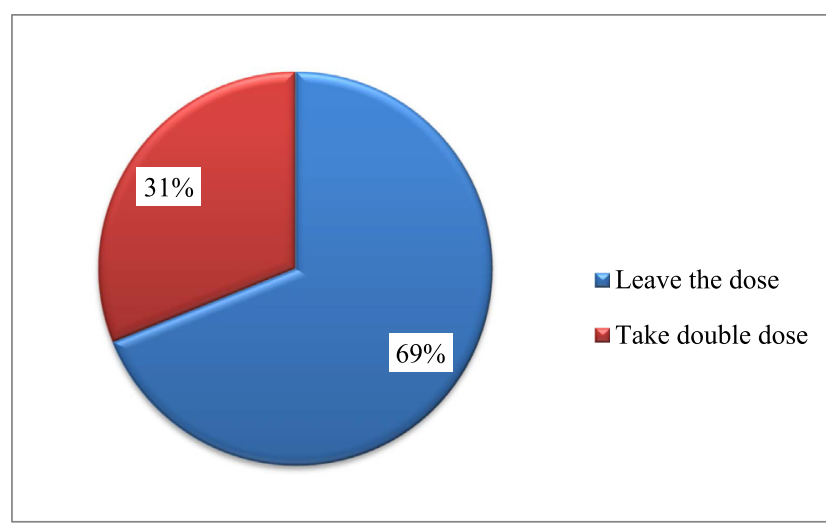

Fig. 3. Public notion on missing a drug dose.

diabetes, hypertension, osteoarthritis, thyroid disorders. $48 \%$ had low adherence to medication, $40 \%$ medium adherence, $12 \%$ high adherence to their medication [Fig. 2]. The test for Analysis of Variance (ANOVA) revealed the variation in cost due to unused medicines among individuals of varying medication adherence was found to be statistically significant. The test result implies that low medication adherence is associated with a higher economic burden due to unused medicine $(p=0.014)$. Families with at least one chronically ill patient tend to leave the Kerala Medical Services Corporation Limited (KMSCL) drugs unused ( $x^{2}=15.426$, $\left.d f=5, p=0.009\right)$. On missing the dose, $69 \%$ of the families left the dose of medicine as opposed to $31 \%$ who took a double dose [Fig. 3]. The medication taking behaviour of the public on missing a drug dose didn't influence the generation of unused drugs $(\mathrm{p}=0.437)$ and the cost due to unused medicines $(\mathrm{p}=0.648) .51 .1 \%$ of the households have admitted that at some point of time, they had left their antibiotic treatment regimen uncompleted. This tendency towards antibiotic treatment presents itself as unused antibiotics $(\mathrm{p}=0.001)$.

\subsection{Storage methods in the households}

A whopping $85 \%$ of the households stored their medicines under ideal conditions, namely-in containers or shelves [Table 1]. The rest of them didn't have any provision for proper storage. The storage of unused antibiotics $(p=0.045)$ and prescription medicines $(p=0.001)$ were under ideal conditions [Table 2].

$76.8 \%$ of the households agreed that they check the expiry of unused medicines every 3 months. 75\% disposed off their unused medicines once in every 3 months. The disposal method of medicines varied considerably. 58\% dumped their medicine either in public places or their backyard, 18\% burnt their medicines in the open, $16 \%$ disposed
Table 1

Respondents' practices and attitudes concerning unused and expired medication handling and disposal.

\begin{tabular}{llll}
\hline Question & Response & N & $\%$ \\
\hline \multirow{2}{*}{ Do you have unused medicines in your house } & Yes & 189 & 54 \\
& No & 161 & 46 \\
How and where do you store unused medicines & Container & 105 & 30 \\
& Shelf & 147 & 42 \\
& Window side & 21 & 6 \\
& Plastic bag & 42 & 12 \\
& Fridge top & 35 & 10 \\
How often do you check the expiry of unused & Every month & 161 & 46 \\
medicines & Every 3 months & 105 & 30 \\
& Every 6 months & 35 & 10 \\
& Yearly & 7 & 2 \\
& Never & 42 & 12 \\
How often do you dispose unused medicines & Every month & 98 & 28 \\
& Every 3 months & 161 & 46 \\
& Every 6 months & 49 & 14 \\
& Yearly & 28 & 8 \\
& Never & 14 & 4 \\
& Dumping & 203 & 58 \\
How do you dispose the unused medicines & Jeevanam & 56 & 16 \\
& Burning & 63 & 18 \\
& Flushing & 7 & 2 \\
& Return to pharmacy & 14 & 4 \\
& Donate & 7 & 2
\end{tabular}

Table 2

The storage conditions of unused antibiotics and prescription drugs.

\begin{tabular}{|c|c|c|c|c|c|c|}
\hline & \multicolumn{4}{|c|}{ Storage conditions } & \multirow[t]{3}{*}{$\chi^{2}$} & \multirow[t]{3}{*}{$\mathrm{p}$} \\
\hline & \multicolumn{2}{|c|}{ Proper } & \multicolumn{2}{|c|}{ Improper } & & \\
\hline & $\mathrm{n}$ & $\%$ & $\mathrm{~N}$ & $\%$ & & \\
\hline Unused Antibiotics & 161 & 86.5 & 25 & 13.5 & 65.581 & 0.0001 \\
\hline Prescription & 160 & 86.4 & 25 & 13.6 & 36.838 & 0.045 \\
\hline
\end{tabular}

through the local waste management system- JEEVANAM. Only a mere $4 \%$ returned their medicine to the pharmacy [Table 1 ].

$53 \%$ of the households had unused medicines with them, out of which, only $31 \%$ were found to be expired or not fit for further use as it lacked proper label information. Summing up, $70.4 \%$ had drug count less than $50,15.6 \%$ had $51-100$ drugs $7 \%$ had $101-150$ drugs, $7 \%$ of the households had unused drug count of more than $150.47 \%$ of households had 1-20 unused antibiotics. KMSCL antibiotics end up unused most of the time $(\mathrm{p}=0.009) .1 .2 \%$ households didn't had any cost due to unused medicines, $24.3 \%$ had cost less than INR 100, $48.9 \%$ had cost between INR 101-500, 18.6\% between INR 501- INR 1000, 7\% had incurred a cost greater than INR1000. The average cost per household was summated to INR 260 per household.

\section{Discussion}

The irrational use of drugs along with impaired regulatory mechanism in handling pharmaceuticals have paved way to a major void in the healthcare system of various developing countries including India. The absence of a waste disposal system specifically designed for pharmaceuticals plays a pivotal role on the attitude of the general public towards drugs. Numerous surveys have been carried out on leftover medicines in the population either by telephonic interview or by sending questionnaires to people, but there are very less number of studies done by home visits with one-on-one personal interview. ${ }^{2,6,7}$ The scarcity of data and studies on the stated matter is evident. The novelty of the study lies in its attempt to outline the qualitative data on medicine taking behaviour, handling, storage and disposal of a community and carrying out the behemoth task of deciphering the same for 


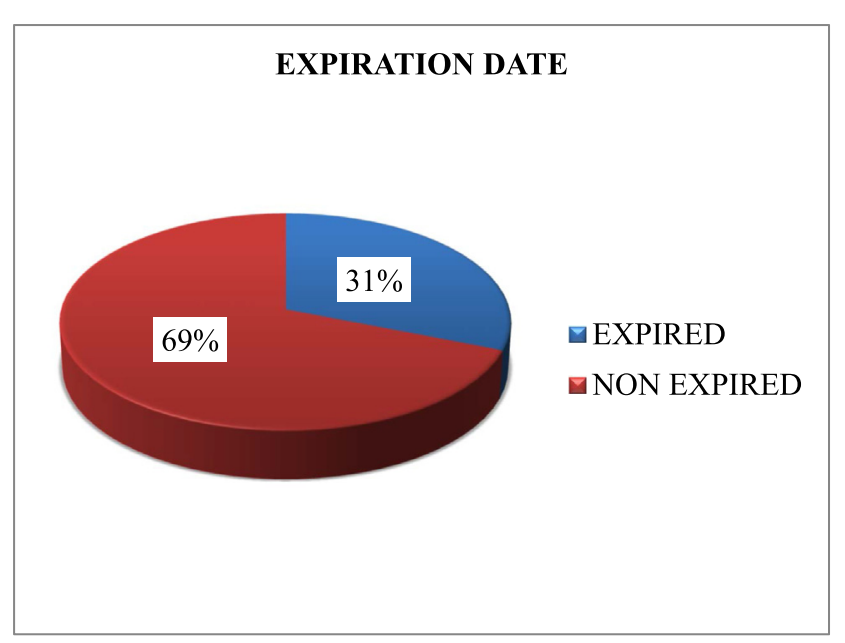

Fig. 4. Status of expired and non expired drugs.

effective policy making. The choice of the study site proves to be far more ideal for the study than once perceived. Perinthalmanna can be described as the hospital hub of Malabar due to the presence of a large number of specialty, super specialty hospitals of both public and private sector. Moreover, the area is thriving with healthcare providers and associated facilities such as laboratories, diagnostic centers, and pharmacies. Hence, the project has its own significance since people have easy access to all the healthcare facilities and comprises of residents representative of a wide range of socio-economic and professional status. Therefore there exists a high possibility for irrational use of medicine leading to a large percentage of leftover medicines accumulating in the households and a plethora of problems associated with it.

The study revealed that a high proportion of households have unused medicines at homes, with an average of 24 medicines per household [Fig. 5]. This number is exorbitantly high compared with the average number of medications stored per household in countries like Sudan, where the mean number of medications per household was 4.4, and Saudi Arabia, where the mean number of medicines was 8.0 per family. ${ }^{4}$ This is not surprising as Perinthalmanna, being a hospital city, the population has unhindered access to hospital services and self medication is high and medicines are kept for re-use in the event of a similar illness or to give it to someone else who might have a similar problem with them.

The total economic burden of the population was calculated to be Rs.72,195.38, which averages at Rs.206 per household. In terms of monetary values, this cost does not have any relevance when compared to the per capita income of Perinthalmanna region (Rs.94,012).But it is

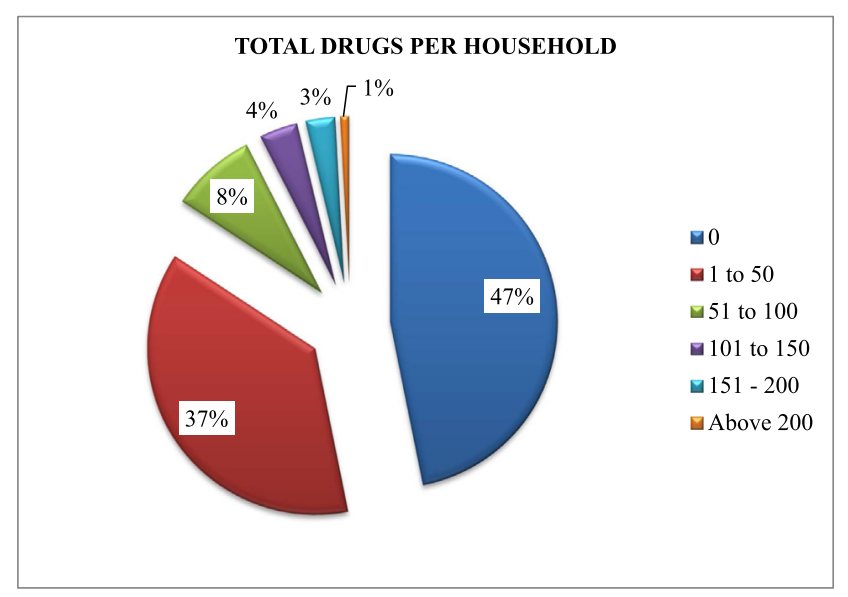

Fig. 5. Total unused drugs per household. to be noted that the cost stated above is only a fraction of what a family spends on treatment, let alone drugs. Moreover, these figures are relatively high, as most of the households dispose off unused medicines every 3-6 months. The cost generated begins to shed light on the unhealthy, luxurious, over the top living standards. The study also revealed that the number of members in a family does not influence the cost due to unused medicine $(\mathrm{p}=0.784)$. The intention of our study was also to expose this scenario to the health care providers and policy makers.

Households where medical professionals lived reported lower occurrence of unused medicines and the cost associated with them were also low compared to households without medical professionals ( $\mathrm{p}=0.043$ ) which was similar to a study conducted by Sebsum et al. ${ }^{5}$ The reason for the skewed data can be pointed out to the fact that medical professionals are well aware of the medication handling techniques and the consequences of unused medicines.

More than $70 \%$ of the population disposes the leftover medicines once in every 3 months, which deserves high appreciation. Though frequency of disposal is appreciable, the methods employed for their disposal is certainly a matter of concern, with dumping (70.6\%) them in the open being principle means of disposal. Despite the frequent disposal of unused medicines, occurrence of unused medicines in households in such an immense amount and their disposal deserves the attention of health care policy makers and professionals.

The study showed that $51.1 \%$ of households did not complete the course of antibiotics. And out of the total number of unused medicines, $14.2 \%$ were antibiotics with an average of 5 antibiotics per households which was lower than a study conducted by Auta B et al., ${ }^{3}$ which reported $33.1 \%$ of unused medicines were antibiotics. This is an indication of the inappropriate use of antibiotics. These antibiotics found can be concluded as a consequence of noncompliance to prescribed antibiotics, over prescription of antibiotics or antibiotics obtained as a result of self medication. The study showed a significant association between the completion of antibiotic regimen and the number of antibiotics that end up unused $(p=0.001)$. This is evident from the high amount of antibiotics reported as leftover medicines by respondents. Inappropriate use and resistance to antibiotics have been reported in developing countries, and this is a major challenge in the treatment of infectious diseases in developing countries where antibiotics are usually obtained without a prescription. ${ }^{7}$ The most common antibiotics that end up unused are amoxicillin, ciprofloxacin, ampicillin, azithromycin, cefixime, metronidazole. An alarmingly high amount of amoxicillin, ampicillin, ciprofloxacin, dicloxacillin were found, which were of KMSCL origin. Despite the stated antibiotics being categorised under access group based on 20th Essential Medicine List by WHO, nevertheless antibiotics as a whole ought to be handled with extreme caution. The storage of antibiotics is satisfactory, in accordance standard guidelines on drug storage $(p=0.045)$.

More than $70 \%$ of the drugs have proper storage whereas the storage of drugs in $30 \%$ of household is highly questionable, but this can be tackled by providing proper awareness on the hazardous effects of drugs caused by improper storage conditions. The condition can be compared to a study conducted in Qatar where $48.2 \%$ of drugs have proper storage. $^{8}$

Out of the total number of households, $49.4 \%$ of them have one or more family members with chronic diseases. Of the reported illnesses, diabetes mellitus $(23.3 \%)$ and hypertension $(12.2 \%)$ was the most common which can be compared to a study conducted by Sebsibe et al. ${ }^{5}$

The study showed that there is no significant association between the presence of a chronically ill family member and the cost of unused medicines ( $p=0.459)$. This is because of the fact that the major source of obtaining medicines for chronic diseases was found to be Kerala Medical Service Cooperation Limited (KMSCL), which are provided free of cost by the government. Since the medicines for chronic diseases are taken on a regular basis, there is no piling up of such medicines unless in case of death or a change in the treatment plan by the physician. 
The medication adherence was measured in chronically ill patients using Moriski Medication Adherence Scale. And the study showed that $48 \%$ of them have very low adherence, followed by medium adherence $40 \%$. The lack of adherence to medication is one of the predominant factors leading to the generation of unused medicines in households $(p=0.014)$. A point to be noted here is that a larger proportion of chronically ill patients obtain their medicines from KMSCL, provided free of cost will lead to the wastage of such medicines, hence indicating lower adherence to medications. In short, adherence to medication is low in special population who need chronic medication.

Solid dosage formulations (tablets and capsules) were the most abundant dosage forms found unused (92\%) with most of the homes keeping tablets between 1 and 50 numbers. This can be due to their ease of administration and acceptability in the community. This can also be attributed to the fact that they are unit dosage forms meant for single use unlike syrups and other dosage forms like ointment, lotion, cream, nasal drops, inhalers. Practically, a single bottle of syrup translates to a week of medication whereas a strip of tablet or capsule with 10 or 15 tablets is needed to last through a week. The results of the study are similar to a study conducted in Uganda. ${ }^{4}$

Interestingly, in this study nearly $80 \%$ of respondents checked the expiry date of medicine once every 3 months, while in contrast in the Indian state of Gujarat many were not aware of the expiry date of medicines. ${ }^{9}$ It is of paramount significance that prior to purchase or use of any medicine, the expiry date must be checked, otherwise it may lead to serious harmful effects. ${ }^{9}$

Out of the total number of unused drugs we documented (8145 numbers), $31 \%$ of drugs were expired medicines and the rest $69 \%$ were non expired medicines [Fig. 4].

The study and its findings generated a series of events resulting in the introduction of a new scheme by the local governing body (Perinthalmanna Municipality) for the effective disposal and reuse of unused medicines from the household and provide awareness on the same. The positive attitude of the population towards pharmaceutical donation, lead to the realisation of a scheme similar to the drug drop off programmes in the developed countries. The unused drugs are collected and are screened based on their reusability. This helps to salvage as much precious, quality medical resource and be redistributed to poor and the destitute patients under professional supervision. The programme attempts to tip the scale of socioeconomic inequalities to balance ideally. It inculcates the next generation with the value of perseverance to attain sustainability; forging sensible and responsible young citizens. The whole system is proposed to be incorporated mainstream by 2019 , the benefits of which can be reaped by nearly 13000 households of the region.

\section{Conclusion}

The results obtained proved the problems related to medicines use among the public are multi dimensional. Provision of proper awareness regarding the problems caused by the unused medicines and the effect it elicits, on the individual, family, community and the environment as a whole can go a long way in the overall health of the public and the generations to follow. The causes due to which unused medicines accumulate can vary, but the threat is ever present. The absence of a proper drug disposal strategy creates a huge void which is hard to miss. Thereby, a multi faceted approach to this problem can be devised by proper planning, creating awareness, and introduction of a proper drug disposal system to ameliorate this condition, not immediately but in a slow and steady pace under proper leadership. A huge majority of the unused drugs are non-expiry medicines and their storage conditions are appreciable. Riding on the back of the quality of the unused drugs, reusing these medications inculcates the minimalistic values into the sustainability of the society.

\section{References}

2. Auta A, Banwat SB, Sariem CN, Shalkur D, Nasara B, Atuluku MO. Medicines in pharmacy students' residence and self-medication practices. J Young Pharm. 2012;4:119-123.

3. Mirza Nazima. BarnaGanguly. Utilization of medicines available at home by general population of rural and urban set up of Western India. J Clin Diagn Res. 2016 Aug;10(8):5-9.

4. Moses Ocan, Bbosa Godfrey S, Paul Waak, Ogwal-Okeng Jasper, CelestinoObua. Factors predicting home storage of medicines in Northern Uganda. BMC Public Health. 2014;14:650-657.

5. FitsumSebsibeTeni, EshetieMeleseBirru, AbdrrahmanShemsuSurur, Belay Assefa, DawitWondimsigegn DessalegnAsmelasheGelayee, ZewdnehShewamene. Pattern and predictors of medicine use among households in Gondar Town, northwestern Ethiopia: a Community-based medicine utilization study. BMC Res Notes. 2017;10:357-365.

6. Lum Elaine PM, Page Katie, Nissen Lisa, Jenny Doust, Graves Nicholas. Australian consumer perspectives, attitudes and behaviours on antibiotic use and antibiotic resistance: a qualitative study with implications for public health policy and practice. BMC Public Health. 2017;17:799-811.

7. Moses Ocan, Ekwaro A, Obuku, Bwanga Freddie, Akena Dickens, Richard Sennono, Ogwal-Okeng Jasper, Obua Celestino. Household antimicrobial self-medication: a systematic review and meta-analysis of the burden, risk factors and outcomes in developing countries. BMC Public Health. 2015;15:742-753.

8. Kheir N, El Hajj MS, AYousif. An exploratory study on medications in Qatar homes. Drug Healthc Patient Saf. 2011;3:99-106.

9. Shah A, Parmar S, Kumkishan A, Mehta A. Knowledge, attitude and practice (KAP) survey regarding the safe use of medicines in rural area of Gujurat. Adv Trop Med Pub Health. 2011;1(2):66-70. 